Pacific Journal of Mathematic 


\title{
AN APPLICATION OF ORTHOGONAL POLYNOMIALS TO RANDOM WALKS
}

\section{Thomas Whitehurst}

\begin{abstract}
If $X_{n}$ is a simple random walk on the nonnegative integers with transition probabilities $P_{i j}^{(k)}=\operatorname{Pr}\left\{X_{n+k}=j \mid X_{n}=i\right\}$, then $P_{i j}^{(k)}$ has an integral representation in terms of a family of orthogonal polynomials and the associated probability distribution function $F(x)$ for these polynomials. The relationship between the distribution $F$, the family of polynomials and the random walk $X_{n}$ is studied. Necessary and sufficient conditions for the support of $F$ to be contained in $[0,1]$ are given.
\end{abstract}

1. Preliminaries. Throughout this paper $X_{n}$ will be a random walk on the integers with transition matrix $P=\left(P_{i j}\right)=\left(\operatorname{Pr}\left\{X_{n+1}=\right.\right.$ $\left.j \mid X_{n}=i\right\}$ ). We shall say that $X_{n}$ is a "simple random walk" if $P_{i j}=0$ whenever $|i-j|>1$, and in this case we set $q_{n}=P_{n n-1}, r_{n}=$ $P_{n n}$, and $p_{n}=P_{n n+1}$. We shall concentrate on simple random walks, $X_{n}$, whose state space is the non-negative integers which we shall henceforth denote by $N_{0}$. Following Karlin and McGregor (1959) we find an integral representation for the transition probabilities $P_{m m}^{(k)}$.

Suppose then that $X_{n}$ is a simple random walk on $N_{0}$. For each state $n \in N_{0}$, we associate a polynomial $Q_{n}(x)$, of degree $n$, defined recursively by

$$
\begin{aligned}
Q_{-1}(x) & =0, Q_{0}(x)=1, \quad \text { and } \\
x Q_{n}(x) & =q_{n} Q_{n-1}(x)+r_{n} Q_{n}(x)+p_{n} Q_{n+1}(x), \quad n \geqq 0 .
\end{aligned}
$$

By the following theorem, due to Favard (1935), we see that the family of polynomials $\left\{Q_{n}(x)\right\}$ defined by (1.1) is orthogonal with respect to a probability distribution $F(x)$.

THEOREM 1.1. Suppose that the family of polynomials $\left\{R_{n}(x)\right\}$ is defined recursively by

$$
\begin{aligned}
R_{0}(x) & =1, R_{1}(x)=x-c_{0}, \quad \text { and } \\
R_{n+1}(x) & =\left(x-c_{n}\right) R_{n}(x)-\lambda_{n} R_{n-1}(x), \quad n \geqq 1,
\end{aligned}
$$

where $c_{n}$ is real and $\lambda_{n+1}>0$ for $n \geqq 0$. Then there is a (probability) distribution function $F(x)$, such that the polynomials $\left\{R_{n}(x)\right\}$ are orthogonal with respect to $F(x)$. That is, $\int_{-\infty}^{\infty} R_{n}(x) R_{m}(x) d F(x)=0$ whenever $n \neq m$. 
THEOREM 1.2. If $\left\{R_{n}(x)\right\}$ is a family of orthogonal polynomials, with $R_{n}(x)$ having degree $n$, and normalized to be monic, then relation (1.2) holds for any three consecutive polynomials, where $c_{n}$ is real and $\lambda_{n+1}>0$, for $n \geqq 0$.

Note that Theorem 1.2 is the converse to Favard's theorem (Theorem 1.1). For a proof and related results see Szegö (1939).

If we write $Q(x)=\left[Q_{0}(x) Q_{1}(x) Q_{2}(x) \cdots\right]^{t}$, then (1.1) is equivalent to $x Q(x)=P Q(x)$, so that $x^{k} Q(x)=P^{k} Q(x)$. In other words, $x^{k} Q_{n}(x)=$ $\sum_{m=0}^{\infty} P_{n m}^{(k)} Q_{m}(x)$. Note that for a simple random walk $X_{n}$, only a finite number of terms on the right side are non-zero. Multiplying both sides of this equation by $Q_{m}(x)$, and exploiting the orthogonality of the family we have

$$
P_{n m}^{(k)}=\pi_{m} \int_{-\infty}^{\infty} x^{k} Q_{n}(x) Q_{m}(x) d F(x),
$$

where $1 / \pi_{m}=\int_{-\infty}^{\infty} Q_{m}^{2}(x) d F(x)$. It can easily be shown, from (1.1), that $\pi_{0}=1$ and $\pi_{m}=\left(p_{0} p_{1} \cdots p_{m-1}\right) /\left(q_{1} q_{2} \cdots q_{m}\right)$.

The support of $F$, Supp $F$, is defined by Supp $F=\{x: F(x+h) \neq$ $F(x-h)$ for all $h \neq 0\}$. If we set $n=m=0$ in (1.3) we see that the support of $F$ is contained in $[-1,1]$ since $P_{n m}^{(k)}$ is a probability. Thus,

$$
P_{n m}^{(k)}=\pi_{m} \int_{-1}^{1} x^{k} Q_{n}(x) Q_{m}(x) d F(x) .
$$

Note that $F(x)$ is uniquely determined since the support of $F$ is contained in a finite interval; hence, the moments uniquely determine the measure.

Karlin and McGregor (1959) took an alternative approach to the above development. That approach was to consider (1.4) as a spectral representation of the linear operator $P$ (our transition matrix) acting on an appropriate Hilbert space. For this reason Karlin and McGregor refer to $F(x)$ in (1.4) as the spectral measure function, SMF, for the random walk $X_{n}$. Using this approach Karlin and McGregor (1959) obtain a representation similar to (1.4) if our simple random walk $X_{n}$ is on $\{0,1,2, \cdots N\}$, or $\{\cdots,-2,-1,0,1,2, \cdots\}$. In the former case (1.1) defines only a finite family of orthogonal polynomials, and the SMF $F$, in (1.4), has only a finite number of support points. In the latter case the representation takes on the form

$$
P_{i j}^{(n)}=\pi_{j} \int_{-1}^{1} x^{n} \sum_{\alpha, \beta=1}^{2} Q_{i}^{(\alpha)}(x) Q_{j}^{(\beta)}(x) d F_{\alpha \beta}(x),
$$

where the $F_{\alpha \beta}(x)$ are distributions on $[-1,1]$, the polynomials $Q_{i}^{(\alpha)}(x)$ 
are defined by

$$
\begin{aligned}
Q_{-1}^{(1)}(x) & =0, Q_{0}^{(1)}(x)=1, Q_{-1}^{(2)}(x)=1, Q_{0}^{(2)}(x)=0, \quad \text { and } \\
x Q_{i}^{(\alpha)} & =q_{i} Q_{i-1}^{(\alpha)}(x)+r_{i} Q_{i}^{(\alpha)}(x)+p_{i} Q_{i+1}^{(\alpha)}(x), \quad(\alpha=1,2) .
\end{aligned}
$$

For $n \geqq 0 \pi_{n}$ is the same as above and

$$
\pi_{-n}=\left(q_{0} q_{-1} q_{-2} \cdots q_{-n+1}\right) /\left(p_{-1} p_{-2} \cdots p_{-n}\right) .
$$

The following results contain some of the essential facts about orthogonal polynomials; for proofs and related results, see Szegö (1939).

Suppose that $F(x)$ is a probability distribution on $[a, b]$ and that $Q_{n}(x)$ is the corresponding family of orthogonal polynomials. Then

THEOREM 1.3. The zeros of $Q_{n}(x)$ are real and distinct, and are located in the interior of the interval $[a, b]$.

THEOREM 1.4. Let $x_{1}<x_{2}<\cdots<x_{n}$ be the zeros of $Q_{n}(x)$; also let $x_{0}=a$ and $x_{n+1}=b$. Then each interval $\left(x_{k}, x_{k+1}\right), 0 \leqq k \leqq n$, contains at least one zero of $Q_{m}(x), m>n$.

THEOREM 1.5. In the open interval $\left(x_{k}, x_{k+1}\right)$, between two consecutive zeros of $Q_{n}(x)$, the function $F(x)$ cannot be constant.

If $F(x)$ is a probability distribution on $[-1,1]$, then one can easily show that there is a family of polynomials $\left\{Q_{n}(x)\right\}$, satisfying (1.1), which are orthogonal with respect to $F(x)$. Furthermore, we may assume that $p_{n}$ and $q_{n+1}$ are positive for $n \geqq 0$. We may also assume that $Q_{n}(1)=1$. This follows from the fact that the zeros of $Q_{n}(x)$ are in $(-1,1)$ so $Q_{n}(1)>0$ for $n \geqq 0$. Since $Q_{n}(1)=1$ we have $q_{n}+r_{n}+p_{n}=1$. Thus, it easily follows that if $F(x)$ has an infinite number of support points, then

THEOREM 1.6. $F(x)$ is the SMF for some simple random walk $X_{n}$ on $N_{0}$ iff

$$
r_{n} / \pi_{n}=\int_{-1}^{1} x Q_{n}^{2}(x) d F(x) \geqq 0 \quad \text { for } \quad n \geqq 0 .
$$

If $F(x)$ has only a finite number of support points, then the simple random walk $X_{n}$ above, is just on $\{0,1,2, \cdots, N\}$.

COROLlaRy 1.7. If $F(x)$ is a probability distribution of $[0,1]$, then $F$ is the SMF for some simple random walk. 
2. The support of the SMF. A sequence of numbers $u=$ $\left(u_{n} ; n \geqq 0\right)$ is a renewal sequence if there exists a sequence $f=$ $\left(f_{n} ; n \geqq 1\right)$ such that $u_{0}=1$ and $u_{n}=\sum_{k=1}^{n} f_{k} u_{n-k}(n \geqq 1)$ where $f_{n} \geqq 0$ and $\sum_{n=1} f_{n} \leqq 1$. An important characterization of renewal sequences is: A sequence $\left(u_{n}\right)$ is a renewal sequence iff $u_{n}=\operatorname{Pr}\left(X_{n}=i \mid X_{0}=i\right)$, $n \geqq 0$, for some Markov chain $X$ and some state $i$. For a proof and related results see Kingman (1972). A renewal sequence $\left(u_{n}\right)$ has period $d$ if and only if $d=$ G.C.D. $\left\{n: u_{n}>0\right\}$. If $d=1,\left(u_{n}\right)$ is said to be aperiodic. If $u$ has period $d$, then ${ }^{d} u=\left(u_{0}, u_{d}, u_{2 d}, \cdots\right)$ is an aperiodic renewal sequence, see Kingman (1972). Thus, it is clear that aperiodic renewal sequences play the major role in the theory of renewal sequences.

ExAmple 2.1. Consider the simple random walk $Y_{n}$ on $Z$ with transition probabilities $P_{i \imath+1}=p_{\imath}, P_{i i-1}=q_{\imath}=1-p_{i}$, and $P_{i j}=0$ otherwise (where $0<p_{i}<1$ ). Note that $v_{n}=\operatorname{Pr}\left(Y_{n}=0 \mid Y_{0}=0\right.$ ) defines a renewal sequence with period 2 . If we start this random walk at an even integer and then take two steps at a time (i.e., we only consider $Y_{2 n}$ ), then we have a "simple" random walk $X_{n}$ on the even integers. (To denote the dependence on the original random walk we shall use the notation $\left.{ }^{2} Y_{n}\right)$. In this case $u_{n}=\operatorname{Pr}\left(X_{n}=\right.$ $\left.0 \mid X_{0}=0\right)=\operatorname{Pr}\left(Y_{2 n}=0 \mid Y_{0}=0\right)$ defines an aperiodic renewal sequence, and in fact $u={ }^{2} v$.

An important type of renewal sequence is the Kaluza sequence, which is defined to be any sequence $u=\left(u_{n} ; n \geqq 0\right)$ such that $0 \leqq$ $u_{n} \leqq u_{0}=1$ and $u_{n}^{2} \leqq u_{n-1} u_{n+1}$ for $n \geqq 1$. Kingman (1972) shows that any Kaluza sequence is always a renewal sequence. By the Schwarz inequality it is clear that

$$
u_{n}=\int_{0}^{1} x^{n} d G(x)
$$

is a Kaluza sequence for any probability distribution $G(x)$ on $[0,1]$. In the above example, if $p_{i} \equiv p, q_{i} \equiv q$, then $u_{n}=\left(\begin{array}{c}2 n \\ n\end{array}\right)(p q)^{n}$ is a Kaluza sequence. Kingman (1972) raises the question of whether this renewal sequence is of the form (2.1). Letac (1977) notes that in the case of $p=q=1 / 2$

$$
u_{n}=\left(\begin{array}{c}
2 n \\
n
\end{array}\right) / 2^{2 n}=\int_{0}^{1}(\cos \pi t)^{2 n} d t=\int_{0}^{1} x^{n} d G(x)
$$

where $d G(x)$ is the measure carried from Lebesque measure on $[0,1]$ by the map $t \mapsto \cos ^{2} \pi t$.

We shall show that the renewal sequence generated in Example 2.1 is always of the form (2.1), even when $p_{i} \not \equiv p$. Furthermore, any 
renewal sequence of the form (2.1) corresponds to a random walk of the form ${ }^{2} Y_{n}$. Following Karlin and McGregor (1959) we shall say that a simple random walk is symmetric if $r_{n} \equiv 0$. The reason for this terminology is that $r_{n} \equiv 0$ iff the SMF $F(x)$ is symmetric about $x=0$.

THEOREM 2.1. Suppose that $F$ is the SMF of the simple random walk $X_{n}$ (on $N_{0}$ ). Then, Supp $F \subset[0,1]$ iff $X_{n}$ is probabilistically the same as ${ }^{2} Y_{n}$, where $Y_{n}$ is a symmetric random walk on $\left\{n / 2: n \in N_{0}\right\}$ which starts at an integer, i.e., $Y_{0}=k, k \in N_{0}$.

Proof. Suppose that Supp $F \subset[0,1]$. Exploiting Theorem 1.6, we define the (symmetric) probability distribution $G(x)$ on $[-1,1]$ by

$$
G(x)=\left\{\begin{array}{lll}
1 / 2\left[1+F\left(x^{2}\right)\right] & \text { if } \quad x \geqq 0 \\
1 / 2\left[1-F\left(x^{2}\right)\right] & \text { if } \quad x<0
\end{array}\right.
$$

$G$ is the SMF of a symmetric random walk, $Y_{n}$, which we may assume to be on $\left\{n / 2: n \in N_{0}\right\}$ taking steps of size $+1 / 2$ and $-1 / 2$. Exploiting (1.4) and the symmetry of $G$ we obtain $\operatorname{Pr}\left\{{ }^{2} Y_{n}=0 \mid{ }^{2} Y_{0}=0\right\}=$ $2 \int_{0}^{1} x^{2 n} d G(x)=\int_{0}^{1} y^{n} d F(y)$. Now ${ }^{2} Y_{n}$ is a simple random walk on $N_{0}$; hence, ${ }^{2} Y_{n}$ has a SMF $H(x)$. Thus, $\operatorname{Pr}\left\{{ }^{2} Y_{n}=0 \mid{ }^{2} Y_{0}=0\right\}=\int_{-1}^{1} x^{n} d H(x)=$ $\int_{0}^{1} x^{n} d F(x)$. However, the moments uniquely determine the measure in this case, so $H(x)=F(x)$. Furthermore, the orthogonal polynomials corresponding to $H(x)$ and $F(x)$ are the same. Hence, the representations given by (1.4) are the same; that is $X_{n}$ is probabilistically the same as ${ }^{2} Y_{n}$.

On the other hand, suppose that $X_{n}$ is probabilistically the same as ${ }^{2} Y_{n}$, where $Y_{n}$ is as given in the statement of the theorem and with $Y_{n}$ having SMF $G(x)$. Since $G(x)$ is symmetric about $x=0$ we have

$$
\operatorname{Pr}\left\{{ }^{2} Y_{n+m}=0 \mid{ }^{2} Y_{m}=0\right\}=\int_{-1}^{1} x^{2 n} d G(x)=2 \int_{0}^{1} x^{2 n} d G(x) .
$$

If $F(x)$ is the SMF for $X_{n}$, then

$$
\operatorname{Pr}\left\{X_{n+m}=0 \mid X_{m}=0\right\}=\int_{-1}^{1} x^{n} d F(x)=2 \int_{0}^{1} x^{2 n} d G(x) .
$$

Setting $y=x^{2}$ and $H(y)=2 G(\sqrt{y})-1$, for $y \geqq 0$, we obtain

$$
\int_{-1}^{1} x^{n} d F(x)=\int_{0}^{1} y^{n} d H(y) .
$$

Again, however, the moments uniquely determine the measure, so that $F(x)=H(x)$, and hence Supp $F \subset[0,1]$. 
As a consequence of Theorem 2.1 we have

CoRollary 2.2. A renewal sequence $u=\left\langle u_{n}\right\rangle$ is of the form (2.1) iff $u_{n}=w_{2 n}$, where $w=\left\langle w_{n}\right\rangle$ is the renewal sequence associated with some symmetric random walk on $N_{0}$.

In the case of a simple random walk on $Z$ we obtain from (1.5) $P_{00}^{(n)}=\int_{-1}^{1} x^{n} d F_{11}(x)$. Hence, the argument of Theorem 2.1 can be extended to this case. Thus, in Corollary 2.2 we may replace $N_{0}$ by $Z$ so that the question raised by Kingman (1972) and answered by Letac (1977) is a special case of this result.

Exploiting Theorem 2.1 we now develop necessary and sufficient conditions for Supp $F \subset[0,1]$, in terms of the coefficients $q_{n}, r_{n}$, and $p_{n}$ in (1.1). In theory one could compute the zeroes of $Q_{n}(x)$ and calculate the support of the SMF, $F$, from them. This procedure is rarely practical because of the complexity of the polynomials, although in some special case $d F(x)$ can actually be calculated (see for example Maki (1967), Karlin and McGregor (1958) or (1959)). In theory one could also use Hausdorff's criteria, in terms of the moments, to test for $\operatorname{Supp} F \subset[0,1]$. Using the fact that $m_{n}=\int_{-1}^{1} x^{n} d F(x)=P_{00}^{(n)}$, we can compute the moments by calculating the probabilities of the various paths. For example $m_{3}=r_{0}^{3}+2 r_{0} p_{0} q_{1}+p_{0} r_{1} q_{1}$. It is clear, however, that this procedure is also not practical. We now give a tractable procedure for determining whether Supp $F \subset[0,1]$.

We shall use the following notation for finite continued fractions:

$$
\begin{aligned}
& \frac{a_{1} \mid}{\mid b_{0}}=a_{1} / b_{0}, \text { and recursively, for } n \geqq 2 \\
& \frac{a_{n} \mid}{\mid b_{n-1}}-\frac{a_{n-1} \mid}{\mid b_{n-2}}-\cdots-\frac{a_{1} \mid}{\mid b_{0}}=a_{n} \div\left[b_{n-1}-\left(\frac{a_{n-1} \mid}{\mid b_{n-2}}-\cdots-\frac{a_{1} \mid}{\mid b_{0}}\right)\right] .
\end{aligned}
$$

To avoid notational difficulties, in the next theorem, we shall assume that our original random walk is on the non-negative even integers, $E_{0}=\left\{2 n: n \in N_{0}\right\}$. We shall use $p_{n}=P_{2 n 2 n+2}, r_{n}=P_{2 n 2 n}$, and $q_{n}=P_{2 n 2 n-2}$.

THEOREM 2.3. Suppose that $F(x)$ is the SMF for the simple random walk $X_{n}$, on $E_{0}$, corresponding to the family of orthogonal polynomials, $\left\{Q_{n}(x)\right\}$, defined by (1.1). Suppose also that $p_{n}+r_{n}+q_{n}=1$ with $p_{n}, q_{n+1}>0$ for $n \geqq 0 \quad\left(q_{0}=0\right)$. Then, Supp $F \subset[0,1]$ iff for $n \geqq 1$

$$
0<h_{n}=\frac{a_{n} \mid}{\mid 1}-\frac{a_{n-1} \mid}{\mid 1}-\cdots-\frac{a_{2} \mid}{\mid 1}-\frac{a_{1} \mid}{\mid 1-p_{0}}<1
$$


where $a_{2 n}=p_{n}$ and and $a_{2 n-1}=q_{n}$.

[Note: We could drop the left inequality since $h_{n}<1$ and $a_{n+1}>$ 0 imply that $h_{n+1}=a_{n+1} /\left(1-h_{n}\right)>0$.]

Proof. We define $\bar{p}_{n}$ and $\bar{q}_{n}$ for $n \geqq 0$ by

$$
\bar{q}_{0}=0, \bar{p}_{1}=p_{0}, \bar{q}_{2 n}=h_{2 n-1}, \bar{p}_{2 n+1}=h_{2 n} \text {, and } \bar{p}_{n}+\bar{q}_{n}=1 \text {. }
$$

If (2.3) is satisfied, then $0<\bar{p}_{n}, \bar{q}_{n}<1$ for $n \geqq 1$. Thus, we may define a symmetric random walk $\bar{X}_{n}$, on $N_{0}$ with transition probabilities $\bar{P}_{n n+1}=$ $\bar{p}_{n}$ and $\bar{P}_{n n-1}=\bar{q}_{n}$. If we start at an even integer, then ${ }^{2} \bar{X}_{n}$ is a random walk on $E_{0}$ with transition probabilities $\overline{\bar{P}}_{2 n 2 n+2}=\bar{p}_{2 n} \bar{p}_{2 n+1}$, $\overline{\bar{P}}_{2 n 2 n-2}=\bar{q}_{2 n} \bar{q}_{2 n-1}$, and $\overline{\bar{P}}_{2 n 2 n}=1-\overline{\bar{P}}_{2 n 2 n-2}-\overline{\bar{P}}_{2 n 2 n+2}$. From the definition of $h_{n}$ and continued fractions we have $\bar{p}_{2 n} \bar{p}_{2 n+1}=\left(1-\bar{q}_{2 n}\right) \bar{p}_{2 n+1}=$ $\left(1-\bar{q}_{2 n}\right) p_{n} /\left(1-\bar{q}_{2 n}\right)=p_{n}$. Thus, $\overline{\bar{P}}_{2 n 2 n+2}=p_{n}$. Similarly, $\overline{\bar{P}}_{2 n 2 n-2}=q_{n}$ and $\overline{\bar{P}}_{2 n 2 n}=r_{n}$. Thus, ${ }^{2} \bar{X}_{n}$ is probabilistically the same as our original random walk $X_{n}$. By Theorem 2.1 Supp $F \subset[0,1]$.

On the other hand, if $\operatorname{Supp} F \subset[0,1]$, then there is a symmetric random walk $Y_{n}$, on $N_{0}$, such that ${ }^{2} Y_{n}$, on $E_{0}$, is probabilistically the same as $X_{n}$. Let $\hat{p}_{n}$ and $\hat{q}_{n}$ represent the transition probabilities of $Y_{n}$. Note that $\hat{r}_{n}=0, \hat{q}_{0}=0$ and $\hat{p}_{n}+\hat{q}_{n}=1$ since $Y_{n}$ is symmetric and ${ }^{2} Y_{n}$ is probabilistically the same as $X_{n}$.

By induction we shall show that $\hat{p}_{n}=\bar{p}_{n}$ and $\hat{q}_{n}=\bar{q}_{n}$, where $\bar{p}_{n}$ and $\bar{q}_{n}$ are given by (2.4). From above we know that $\hat{p}_{0}=1=\bar{p}_{0}$ and $\hat{q}_{0}=1=\bar{q}_{0}$. Assume then that $\hat{p}_{n}=\bar{p}_{n}$ and $\hat{q}_{n}=\bar{q}_{n}$ for $n \leqq m$. If $m$ is even, then

$$
\begin{aligned}
p_{m / 2} & =\operatorname{Pr}\left\{X_{k+1}=m+2 \mid X_{k}=m\right\}=\operatorname{Pr}\left\{{ }^{2} Y_{k+1}=m+2 \mid{ }^{2} Y_{k}=m\right\} \\
& =\hat{p}_{m} \hat{p}_{m+1}=\bar{p}_{m} \hat{p}_{m+1} .
\end{aligned}
$$

Thus, $\hat{p}_{m+1}=p_{m / 2} \div \bar{p}_{m}=p_{m / 2} \div\left(1-\bar{q}_{m}\right)=\bar{p}_{m+1}$ from (2.3) and (2.4). Similarly, if $m$ is odd, then $\hat{q}_{m+1}=q_{(m+1) / 2} \div \bar{q}_{m}=\bar{q}_{m+1}$. Since $\hat{q}_{n}+\hat{p}_{n}=$ $1=\bar{q}_{n}+\bar{p}_{n}$, we have in either case $\hat{p}_{m+1}=\bar{p}_{m+1}$ and $\hat{q}_{m+1}=\bar{q}_{m+1}$. Thus, $\hat{p}_{n}=\bar{p}_{n}$ and $\hat{q}_{n}=\bar{q}_{n}$. It is clear that $0<\hat{p}_{n}, \hat{q}_{n}<1$ for $n \geqq 1$, so from (2.4) we see that $0<h_{n}<1$ for $n \geqq 1$.

In many cases the continued fractions in (2.3) will not be difficult to compute since $h_{n+1}=a_{n+1} /\left(1+h_{n}\right)$.

EXAMPLE 2.2. Suppose that $p_{0}=1 / 2=r_{0}$ and for $n \geqq 1 r_{n}=1 / 2$ and $p_{n}=q_{n}=1 / 4$. From (2.3) we see that $h_{n}=1 / 2$ for $n \geqq 1$. Thus, by Theorem 2.3, we see that the SMF corresponding to this random walk is supported by $[0,1]$.

EXAMPLE 2.3. Suppose that $p_{0}=1 / 3$ and $r_{0}=2 / 3$, and for $n \geqq$ 
$1, r_{n}=p_{n}=q_{n}=1 / 3$. From (2.3) $h_{1}=1 / 2, h_{2}=2 / 3$, but $h_{3}=1$. Thus, the SMF corresponding to this random walk is not supported by $[0,1]$.

3. Some special cases. Consider a family of polynomials $\left\{R_{n}(x)\right\}$, defined by (1.2), which are orthogonal with respect to $F(x)$. Let $X$ be the set of zeros; i.e., $X=\left\{x: R_{n}(x)=0\right.$ for some $\left.n \geqq 1\right\}$. From Blumenthal (1898) we have

THEOREM 3.1. Suppose that $c_{n}$ and $\lambda_{n}$ converge to $c$ and $\lambda$ (finite) respectively. Then the set $X$ is dense in $[\sigma, \tau]$ where $\sigma=c-2 \lambda^{1 / 2}$ and $\tau=c+2 \lambda^{1 / 2}$; hence, the Supp $F$ is dense in $[\sigma, \tau]$. Furthermore, $d F$ consists only of a countable number of atoms outside of $[\sigma, \tau]$.

For a proof and related results see Chihara (1968).

CoRollary 3.2. If $X_{n}$ is a simple random walk on $N_{0}$ with SMF $F$, then $d F$ is purely atomic if $r_{n} \rightarrow 1$.

Proof. The corresponding family of orthogonal polynomials $\left\{Q_{n}(x)\right\}$ is defined by (1.1). If we normalize $Q_{n}(x)$ to be monic, then the normalized polynomials satisfy (1.2) with $c_{n}=r_{n}$ and $\lambda_{n}=p_{n-1} q_{n}$. Since $q_{n}+r_{n}+p_{n}=1$, we see that $\lambda_{n} \rightarrow 0$. so $\sigma=\tau=1$.

Still assuming that $X_{n}$ has SMF $F$ we have

TheOREM 3.3. If Supp $F \subset[0,1]$ and $r_{n} \rightarrow 0$, then $d F$ is purely atomic.

Proof. We may assume that $q_{n}+r_{n}+p_{n}=1$ since normalizing $Q_{n}(x)$ so that $Q_{n}(1)=1$ does not affect $r_{n}$. For $n \geqq N(\varepsilon) r_{n}<\varepsilon$. By Theorem 2.3 we have $h_{2 n}=p_{n} /\left(1-h_{2 n-1}\right)<1$ so $p_{n}+h_{2 n-1}<1$ and $h_{2 n-1}=q_{n} /\left(1-h_{2 n-2}\right)>q_{n}$.

Thus, for $n \geqq N(\varepsilon), \quad 1-\varepsilon<p_{n}+q_{n}<p_{n}+h_{2 n-1}<1$. Hence, $0<h_{2 n-1}-q_{n}<\varepsilon$; but $h_{2 n-1}-q_{n}=q_{n}\left[h_{2 n-2} /\left(1-h_{2 n-2}\right)\right]$. Also $p_{n-1}=$ $h_{2 n-2}\left(1-h_{2 n-3}\right)<h_{2 n-2}<\left[h_{2 n-2} /\left(1-h_{2 n-2}\right)\right]$. Therefore, $0<\lambda_{n}=q_{n} p_{n-1}<$ $q_{n}\left[h_{2 n-2} /\left(1-h_{2 n-2}\right)\right]<\varepsilon$ for $n \geqq N(\varepsilon)$. Hence $\lambda_{n} \rightarrow 0$ and $d F$ is purely atomic.

For general families of orthogonal polynomials $\left\{R_{n}(x)\right\}$ defined by (1.2) with $\operatorname{Supp} F \subset[a, b]$ we have

CoRollaRY 3.4. If $c_{n} \rightarrow a$ or $c_{n} \rightarrow b$, then $d F$ is purely atomic.

Proof. Starting with (1.2) we set $Q_{n}(x)=R_{n}(y) / R_{n}(b)$ where $x=$ $(y-a) /(b-a)$. This new family of polynomials $\left\{Q_{n}(x)\right\}$ is orthogonal 
on $[0,1]$ with SMF $F$ translated to $[0,1]$. The family $\left\{Q_{n}(x)\right\}$ satisfies (1.1) with $q_{n}=\lambda_{n} t_{n-1} /(b-a) t_{n}, r_{n}=\left(c_{n}-a\right) /(b-a)$, and $p_{n}=t_{n+1} /(b-a) t_{n}$ where $t_{n}=R_{n}(b)$ which is positive by Theorem 1.3. Since $Q_{n}(1)=1$ we have $q_{n}+r_{n}+p_{n}=1$ so we may apply the previous two results to this family. If $c_{n} \rightarrow a$ or $c_{n} \rightarrow b$ then $r_{n} \rightarrow 0$ or $r_{n} \rightarrow 1$. Thus, the original measure $d F$ is purely atomic.

Note that if $\operatorname{Supp} F=[0,1]$ and $p_{n}, q_{n}$ and $r_{n}$ converge to $p, q$ and $r$ respectively, then a necessary condition for $F$ to be absolutely continuous is that $r=1 / 2$ and $p=q=1 / 4$. This follows immediately from Theorem 3.1.

ACKNowledgement. The author wishes to thank Professor Daniel Maki for his helpful suggestions and discussions concerning this paper.

\section{REFERENCES}

1. O. Blumenthal, Über die Entwicklung einer wilkürlichen Funktion..., Inaugural Dissertation, (1898), Göttingen.

2. T. S. Chihara, Orthogonal polynomials whose zeros are dense in intervals, J. Math. Anal. and Appl., 24, No. 2, (1968), 362-371.

3. J. Favard, Sur les polynomes de Tchebicheff, C. R. Acad. Sci. Paris, 200 (1935), 2052-2053.

4. S. Karlin and J. McGregor, Linear growth, birth and death processes, J. Math. Mech., 7 (1958), 643-662.

5. — Random Walk, Illinois J. Math., 3 (1959), 66-81.

6. J. F. C. Kingman, Regenerative phenomena, (1972), London, Wiley.

7. G. Letac, Recurrence for products of renewal sequences, Ann. Probability, 5, No.

4, (1977), 591-594.

8. D. Maki, On constructing distribution functions; a bounded denumerable spectrum with $n$ limit points, Pacific J. Math., 22 (1967), 431-452.

9. G. Szegö, Orthogonal polynomials, Amer. Math. Soc. Colloq. Publ., 23 (1939), New York.

Received February 4, 1980.

Florida International University

MIAMI, FL 33199 



\section{PACIFIC JOURNAL OF MATHEMATICS}

\section{EDITORS}

DONALD BABBITT (Managing Editor)

University of California

Los Angeles, California 90024

\section{Hugo Rossi}

University of Utah

Salt Lake City, UT 84112

C. C. MOore and Arthur AGuS

University of California

Berkeley, CA 94720
J. DugundJI

Department of Mathematics University of Southern California Los Angeles, California 90007

R. FinN and J. Milgram Stanford University Stanford, California 94305

ASSOCIATE EDITORS
R. ARENS
E. F. BECKENBACH
B. H. NeumanN
F. WOLF
K. YOSHIDA

\section{SUPPORTING INSTITUTIONS}

UNIVERSITY OF ARIZONA

UNIVERSITY OF BRITISH COLUMBIA

CALIFORNIA INSTITUTE OF TECHNOLOGY

UNIVERSITY OF CALIFORNIA

MONTANA STATE UNIVERSITY

UNIVERSITY OF NEVADA, RENO

NEW MEXICO STATE UNIVERSITY

OREGON STATE UNIVERSITY
UNIVERSITY OF OREGON

UNIVERSITY OF SOUTHERN CALIFORNIA

STANFORD UNIVERSITY

UNIVERSITY OF HAWAII

UNIVERSITY OF TOKYO

UNIVERSITY OF UTAH

WASHINGTON STATE UNIVERSITY

UNIVERSITY OF WASHINGTON 


\section{Pacific Journal of Mathematics}

Vol. 99, No. $1 \quad$ May, 1982

Mariano Giaquinta, Jindrich Necas, O. John and J. Stará, On the

regularity up to the boundary for second order nonlinear elliptic systems . . 1

Siegfried Graf, Realizing automorphisms of quotients of product $\sigma$-fields . . 19

Alfred Washington Hales and Ernst Gabor Straus, Projective colorings . . . 31

Sandra Hayes, The weak Nullstellensatz for finite-dimensional complex

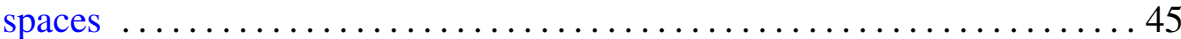

Gerald Norman Hile and Murray Harold Protter, The Cauchy problem

and asymptotic decay for solutions of differential inequalities in Hilbert

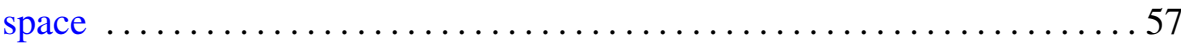

Robert D. Little, Projective space as a branched covering with orientable

branch set ......................................... 89

Jaroslav Mach, On the proximinality of Stone-Weierstrass subspaces . . . . . 997

John C. Morgan, II, On product bases ...................... 105

K. Balakrishna Reddy and P. V. Subrahmanyam, Altman's contractors

and fixed points of multivalued mappings . .................. 127

James Ted Rogers Jr., Decompositions of homogeneous continua . . . . . . . 137

Ahmed Ramzy Sourour, Characterization and order properties of

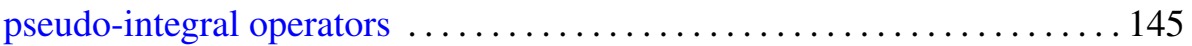

Robert Moffatt Stephenson Jr., Pseudocompact and Stone-Weierstrass

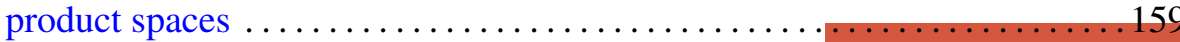

Bruce Stewart Trace, On attaching 3-handles to a 1-connected

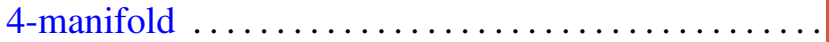

Akihito Uchiyama, The construction of certain BMO functions and the corona problem

Thomas Alva Whitehurst, An application of orthogonal polynomials to random walks ..............................

David J. Winter, Root locologies and idempotents of Lie and nonassociative algebras

William Robin Zame, The classification of uniform algebras on plane domains 